International Journal of Pattern Recognition and Artificial Intelligence

Vol. 30, No. 9 (2016) 1660011 (16 pages)

(C) The Author(s)

DOI: $10.1142 /$ S0218001416600119

\title{
Redundancy Reduction Algorithms in Rule-Based Knowledge Bases
}

\author{
Yongjie Zhang* and Ansheng Deng ${ }^{\dagger}$ \\ University Department \\ Dalian Maritime University, Dalian \\ Dalian, Liaoning, P. R. China \\ *zhangyongiie523@sina.com \\ †ashdeng@dlmu.edu.cn
}

Received 26 November 2015

Accepted 13 September 2016

Published 19 October 2016

\begin{abstract}
Redundancy rules in knowledge bases will affect the reasoning process of knowledge bases. And they will take up a lot of unnecessary memory space. So the notions of redundancy rules are briefly introduced. Meanwhile they are classified into four types. This paper studies the redundancy rules based on propositional logic and presents the reduction algorithms of four kinds of redundancy rules. They are equivalent redundancy rules, implication redundancy rules and cycle redundancy rules in explicit redundancy rules and condition redundancy rules in implicit redundancy rules. The reduction in this paper optimizes the structure of rule-based knowledge bases. And it also improves the efficiency of time and space of the reasoning on knowledge bases.
\end{abstract}

Keywords: Rule-based knowledge bases; explicit redundancy rules; implicit redundancy rules; reduction.

\section{Introduction}

With the popularity of the rapid developing of computer technology and networks, artificial intelligence technology has penetrated into all walks of life of the computer applications and networks. And it will promote the transformation of these industries and the computer software industry itself.

As a very important part of artificial intelligence, the knowledge base is a knowledge-based problem solving system. ${ }^{1}$ When it shows expert problem-solving ability it becomes an expert system. ${ }^{2}$

\footnotetext{
$\dagger$ Corresponding author.
}

This is an Open Access article published by World Scientific Publishing Company. It is distributed under the terms of the Creative Commons Attribution 4.0 (CC-BY) License. Further distribution of this work is permitted, provided the original work is properly cited. 
The establishment of the rule base is very time consuming. And there are some similar rule bases. So integrating the similar existing rule bases can be a way to solve this problem. But this method can lead to the existing of redundancy rules. ${ }^{6}$ The redundancy rules in knowledge bases make the system inefficient and lead to a low space utilization. There may be other negative consequences as well such as high cost for maintenance. So, the avoidance of redundancy rules is an important issue that must be addressed in the organization and management of knowledge bases.

The maintenance of knowledge bases ${ }^{4}$ includes the rule addition, modification and rules reduction. ${ }^{5}$ There is no generally accepted method with high efficiency for reduction of redundancy rules. Most people tend to detect redundancy rather than to reduct redundancy, see Refs. 7 and 11 for examples. But for a known rule-based knowledge base, its rules already exist, redundancy already exists too. And the number of redundancy rules is in a certain size. This affects the reasoning and computation speed in the knowledge bases badly, and consumes unnecessary memory.

For these reasons, this paper studies the redundancy rules in proposition logic ${ }^{9}$ and gives redundancy reduction algorithms and their implementation. There is a paper that do some research in the quadratic programming to decrease the time complexity of the algorithm. ${ }^{8}$ And this paper also find a way similar to that which will be discussed more fully later in this paper.

\section{Rule-Based Knowledge Bases}

Usually a rule-based knowledge base consists of six components which are the knowledge bases, the reasoning engine, the integrated database, the explanation interface, the knowledge acquisition program and the human machine interface. This paper is aimed at the reduction of the redundancy rules. Thus only the knowledge base is taken into study.

The knowledge base is the storage of all for the knowledge in the rule-based knowledge base. Its main functions are to store the rules and maintain the knowledge in the knowledge base.

The rules in the knowledge base are classified into two types. One is the facts which are the rules with premises but without conclusions. The other is called interference rules which are the rules with both premises and conclusions. And the interference rule (simply called rule) is the main part that will be reducted.

\section{Redundancy Rules}

Redundancy rule is an important concept in the data mining and meanwhile it is also very important in the firewall politics, see Refs. 3 and 12 for examples. And in the rule-based knowledge base the redundancy rule is also an important concept as well so this paper will explain the concept in detail. Rule reduction is deleting rules from a knowledge base without narrowing or enlarging the set that is derived from the former knowledge base. ${ }^{13}$ 
The redundancy rules can be classified into four classes. ${ }^{13}$ They are explicit redundancy rules, implicit redundancy rules, similarity redundancy rules and abstract redundancy rules. In the following parts this paper will introduce the explicit redundancy rules, and implicit redundancy rules in detail. As to the similarity and abstract redundancy rules this paper just gives the notions of both of them because of the prediction involved. The reduction of these should be discussed in the future.

\subsection{Explicit redundancy rules}

Definition 1. The explicit redundancy rules are the rules that can be distinguished by the external form of rules.

From the definition above, the explicit redundancy rules can be easily judged. And it is not necessary to check the internal form which means the conditions. The explicit redundancy rules are classified into four classes which are equivalent redundancy rules, cycle redundancy rules, contradiction redundancy rules and implication redundancy rules.

Definition 2. Equivalent redundancy rules refer to the rules with the equivalent premises and conclusions.

For example there are two rules. One of the rule is $P \rightarrow Q$. And the another rule is $R \rightarrow S$. $P, Q, R$ and $S$ are propositions here. The proposition $P$ is equivalent to $R$. And the proposition of $Q$ is equivalent to $S$. Both the premises and the conclusions of the rules are equivalent so the two rules are equivalent rules.

Definition 3. Cycle redundancy rules are a sequence of rules. The conclusion of the first rule is the equivalent to the premise of the second rule. The conclusion of the second rule is the equivalent to the premise of the third rule. And so on the conclusion of the $n-1$ rule is equivalent to the premise of the $n$ rule. And the conclusion of the $n$ rule is equivalent to the premise of the first rule.

For example a sequence of rules is in the following form: $P_{1} \rightarrow P_{2}, P_{2} \rightarrow P_{3}$, $P_{3} \rightarrow P_{4}, \ldots, P_{i-1} \rightarrow P_{i}, \ldots, P_{n-1} \rightarrow P_{n}, P_{n} \rightarrow P_{1}$. $P_{i}$ is a proposition here. Then these rules are cycle rules.

Definition 4. Contradictory conclusions are two conclusions that the names of the propositions in the two conclusions are the same. But if the propositions in the first conclusion are positive then propositions in the other are negative and they apply to the reverse. In a word the propositions in the two conclusions are contradictory.

For example one of the conclusions is $P$ and the other conclusion is $\neg P$. $P, \neg P$ are propositions here.

Definition 5. Contradiction redundancy rules are the rules with the same premise but the contradictory conclusions. 
Definition 6. Implication redundancy rules are two rules with the same conclusion and the premise of one rule is contained by the other rule's conclusion.

For example one of the rules is $P, Q \rightarrow R$ and the other rule is $P \rightarrow R . P, Q$ and $R$ are propositions here. The two rules there are contradiction redundancy rules.

\subsection{Implicit redundancy rules}

As explained before, the explicit redundancy rules are the rules that can be distinguished by the external form. From the literal sense, the implicit redundancy rules can be defined in the similar way. In this section, the definition and the classifications of it will be presented.

Definition 7. Implicit redundancy rules are the rules that cannot be distinguished by the external form.

The implicit redundancy rules can be classified into three classes. The three classes are condition redundancy rules, unnecessary condition redundancy rules and transmition redundancy rules.

Definition 8. Condition redundancy rules are the rules with the specific premise. There are conditions in the premise that do not exist in the rule database and do not exist in the conclusion of other rules either.

For example there is a rule database, which has five rules. The five rules are $P, Q$, $P \rightarrow R, T \rightarrow S$ and $P, Q \rightarrow W$. In these five rules, the premise of rule $T \rightarrow S$ is $T$. But this premise does not exist in the rule database and it does not exist in the conclusion of other rules either. So the rule $T \rightarrow S$ is a condition redundancy rule.

Definition 9. Unnecessary condition redundancy rules are the rules with the same conclusion but have the contradict conditions in the premises.

For example there are two rules in the following form: $P, R \rightarrow Q$ and $P, \neg R \rightarrow Q$. $P, Q$ and $R$ are propositions here. The proposition $R$ here is useless. So this paper call the two rules unnecessary condition redundancy rules.

Definition 10. Transmition redundancy rules are two sequences of rules. The first rules of the two sequences have the same premise and the conclusions of the last rules in the two sequences are equivalent.

\subsection{Similarity redundancy rules}

Definition 11. The similar literals are two literals with the same predication. But they can carry different items.

Definition 12. Similarity redundancy rules are the rules whose premises and concludes consist of similar literals. 
For example the two rules are $P\left(t_{1}\right) \rightarrow Q\left(t_{1}\right)$ and $P\left(t_{2}\right) \rightarrow Q\left(t_{2}\right) . P\left(t_{1}\right), Q\left(t_{1}\right), P\left(t_{2}\right)$ and $Q\left(t_{2}\right)$ are predications here. And $t_{1}$ and $t_{2}$ are items here. These rules are similarity redundancy rules.

\subsection{Abstract redundancy rules}

Definition 13. Abstract redundancy rules are two rules. The predications in the two rules are the same. But the items in one of the rules are abstract such as variables. And the items in the other rule are specific such as constants.

For example the two rules are $P(x) \rightarrow Q(x)$ and $P(a) \rightarrow Q(a) . P(x), Q(x), P(a)$ and $Q(a)$ are predications here. And $x$ and $a$ are items here. The $a$ is a constant which means the item is specific. The $x$ is a variable which is abstract. The rule $P(x) \rightarrow$ $Q(x)$ is similarity redundancy rule.

\section{Reduction Algorithm of Redundancy Rules Based on Knowledge Bases}

The existence of redundancy rules will affect the storage and the efficiency of the rule database. When the scale of the knowledge database is huge, the reasoning in the database will produce some new rules. And if the scale of the knowledge database is huge already, the database after the reasoning can be too huge so that there may be the risk of state space explosion. What is more, the existence of redundancy rules will cause the low speed of reasoning and the low efficiency of time and space. Even worse, the inference may fall into a dead cycle because of the cycle redundancy rules.

Since the reduction of the redundancy rules becames quite necessary. The reduction algorithm of redundancy rules based on knowledge base is proposed here.

The similarity redundancy rules and abstract redundancy rules involve the concept of item. The item is a concept in predicate logic, and the reduction in predicate logic is too difficult. That should be discussed in the future. For these reasons, this paper studies the redundancy rules in proposition logic. So this paper focus on the reduction of explicit redundancy rules and implicit redundancy rules.

Explicit redundancy rules account for a larger proportion of redundancy rules. ${ }^{10}$ The existence of explicit redundancy rules does not influence other rules. Meanwhile the judge of implicit redundancy is difficult and the reduction of implicit redundancy rules is always related to the system efficiency and other factors. The Contradiction redundancy rules is an independent research. It can be dealt by revision in knowledge base. So this paper firstly give the reduction algorithms for equivalent redundancy rules and implication redundancy rules in explicit redundancy rules. And this paper present the reduction algorithm for condition redundancy rules which is implicit redundancy rules. These three kinds of redundancy rules are relatively simple. Then with more efforts the algorithm for cycle redundancy rules in explicit redundancy rules is conducted. 
The reduction of the equivalent redundancy rules and the implication redundancy rules can use the same way. So the reduction for these two kinds of redundancy rules are classified into two classes. And these rules can be reducted using the similar way.

Based on the above reasons the reduction of explicit and implicit redundancy rules should be the focus of the redundancy rules reduction.

A rule is the minimum element in the set of the reduction process. The premise and the conclusion are the sets of variables or parameters. ${ }^{1}$

In the rule-based knowledge base the premise of one of the rule can be described as a set consist of propositions. The form of the set is defined as:

$$
C_{i}=\left\{P_{1}, \ldots, P_{n}\right\} \text {. }
$$

The $P_{j}$ is the $j$ proposition in the premise $C_{i}$. And $P_{j}$ is denoted as $\left(P, n_{P}\right) . P$ is the proposition and $n_{P}$ is the clause position of the proposition in the premise. The $n$ in the definition is the number of propositions in the premise $C_{i}$.

In the rule-based knowledge base the conclusion of one of the rule can be described as a set consist of propositions too. So the conclusion can be defined in the similar way of the definition of the premise. The conclusion can be defined in the following way:

$$
D_{i}=\left\{Q_{1}, \ldots, Q_{m}\right\}
$$

The definition of the conclusion $D_{i}$ is similar to the premise $C_{i}$. By the definition of premise, the set of the premise can be described here. The form of set is defined as the following way:

$$
C=\left\{C_{1}, \ldots, C_{r_{1}}\right\} .
$$

And in the same way, the conclusion set can be defined as follows:

$$
D=\left\{D_{1}, \ldots, D_{r_{2}}\right\}
$$

With all the definitions above, the rule base can be described as follows:

$$
R=\{C, D\} .
$$

\subsection{Reduction algorithm for equivalent and implication redundancy rules}

According to the previous analysis the reduction algorithms for equivalent redundancy rules and implication redundancy rules in the type of explicit redundancy rules will be introduced firstly here.

Both of the algorithms are divided into two parts, the first part is aimed to build a virtual sorting. And the second part is to actualize the reduction based on the virtual sorting in the first part. The first part is the highlights of the two algorithms. And the second part is the key point of the algorithms.

Before the virtual sorting, the creation of an intermediate structure is necessary. And the intermediate structure is the unit to be used in the virtual sort algorithm. 


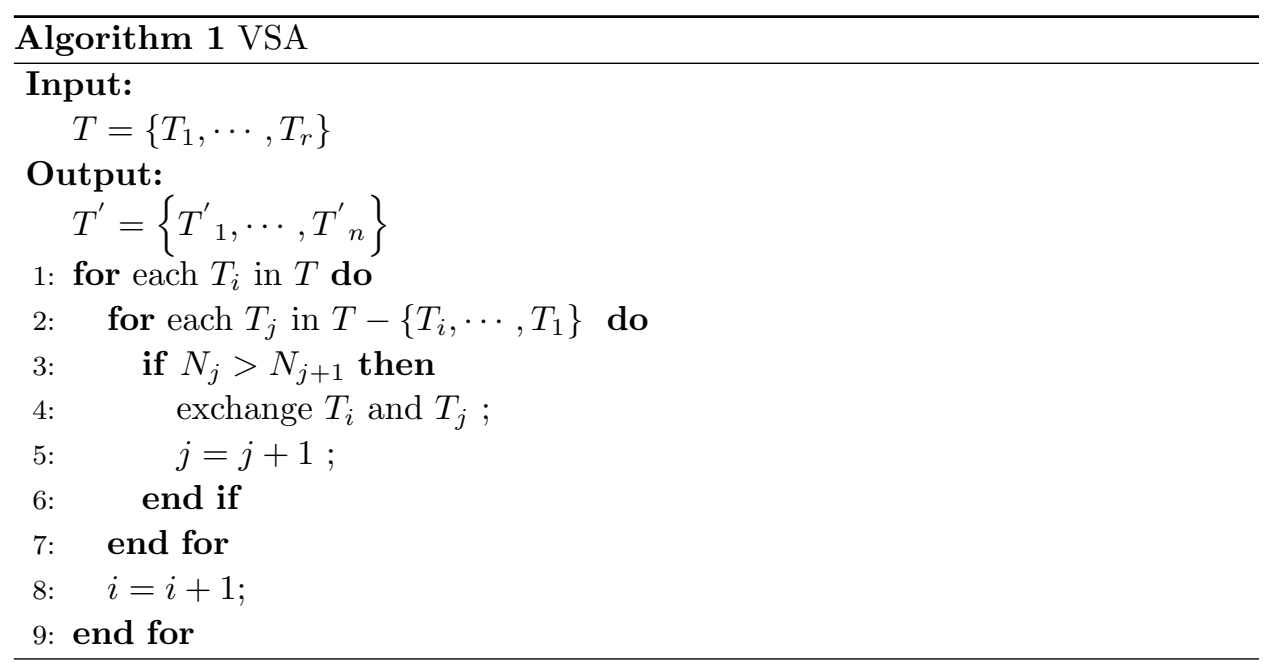

The intermediate structure can be defined as follows:

$$
T=\left\{T_{1}, \ldots, T_{r}\right\}
$$

Every element in the set is defined in the following way:

$$
T_{i}=\left\{I_{1}, N_{i}\right\} .
$$

$I_{i}$ is the position of the $i$ rule in the rule base. $I_{i}$ is the number of the propositions in the $i$ rule.

With the intermediate structure been built above the sorting of $R$ can be transferred into the sorting of $T$. Based on the notions above, the algorithm of the virtual sorting algorithm can be presented here (VSA, Algorithm 1). The input is the original intermediate structure gained by the rules set, and the output is the intermediate structure after the sorting. The sorting is conducted in ascending order of standard by $N$.

After the sorting, the sorting intermediate structure $T^{\prime}$ can be used to conduct our reduction algorithm for equivalent redundancy rules (ERRRA, Algorithm 2). The reduction algorithm for equivalent redundancy rules is presented here. The input is the original rule base, and the output is the rule base after the reduction. The fourth and fifth step in the ERRRA is matching steps, this algorithm just simply matches every proposition in the two premise sets and the two conclusion sets to judge whether they are equivalent to each other or not.

With the sorting algorithm, the sorting intermediate structure $T^{\prime}$ can be used to conduct the reduction algorithm for implication redundancy rules (IRRRA, Algorithm 3). The reduction algorithm for implication redundancy rules is presented here. The input is the original rule base, and the output is the rule base after the reduction. The fourth step in the IRRRA is the matching step, this algorithm just simply matches every proposition in the two premise sets to judge whether they are 

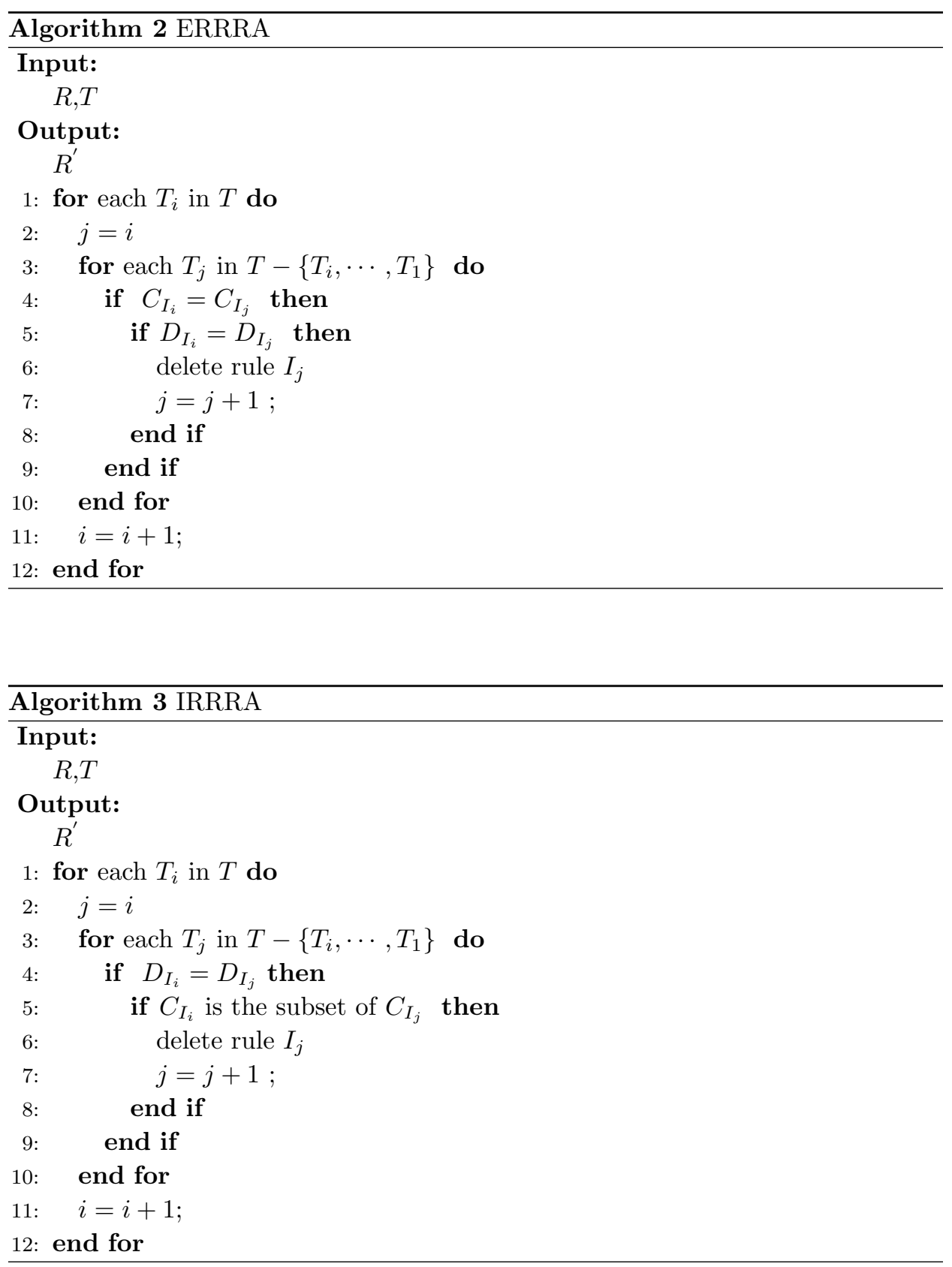
equivalent to each other or not. And in the fifth step this algorithm uses the same way to text whether $C_{I_{j}}$ is the subset of $C_{I_{i}}$.

\subsection{Reduction algorithm for condition redundancy rules}

The reduction for condition redundancy rules of the implicit redundancy rules is not related to the number of propositions. So there is no need to concern about the number of propositions. Thus the virtual sorting is not necessary for this algorithm.

So the sorting algorithm is not necessary here. This paper just simply compares every proposition in premise of every rules with the proposition in the conclusion of other rules. If there is a match for every proposition of a rule, that means this rule is not condition redundancy rule. If there is no match for every proposition of a rule, this rule is a condition redundancy rule. Then this rule will be deleted. The algorithm for the reduction for condition redundancy rules (CRRRA, Algorithm 4) is presented here. The input is the original rule base, and the output is the rule base after the reduction.

\subsection{Reduction algorithm for cycle redundancy rules}

In order to make the reduction algorithm for cycle redundancy rules more easily, a new structure of premises and a new structure of conclusions are conducted here.

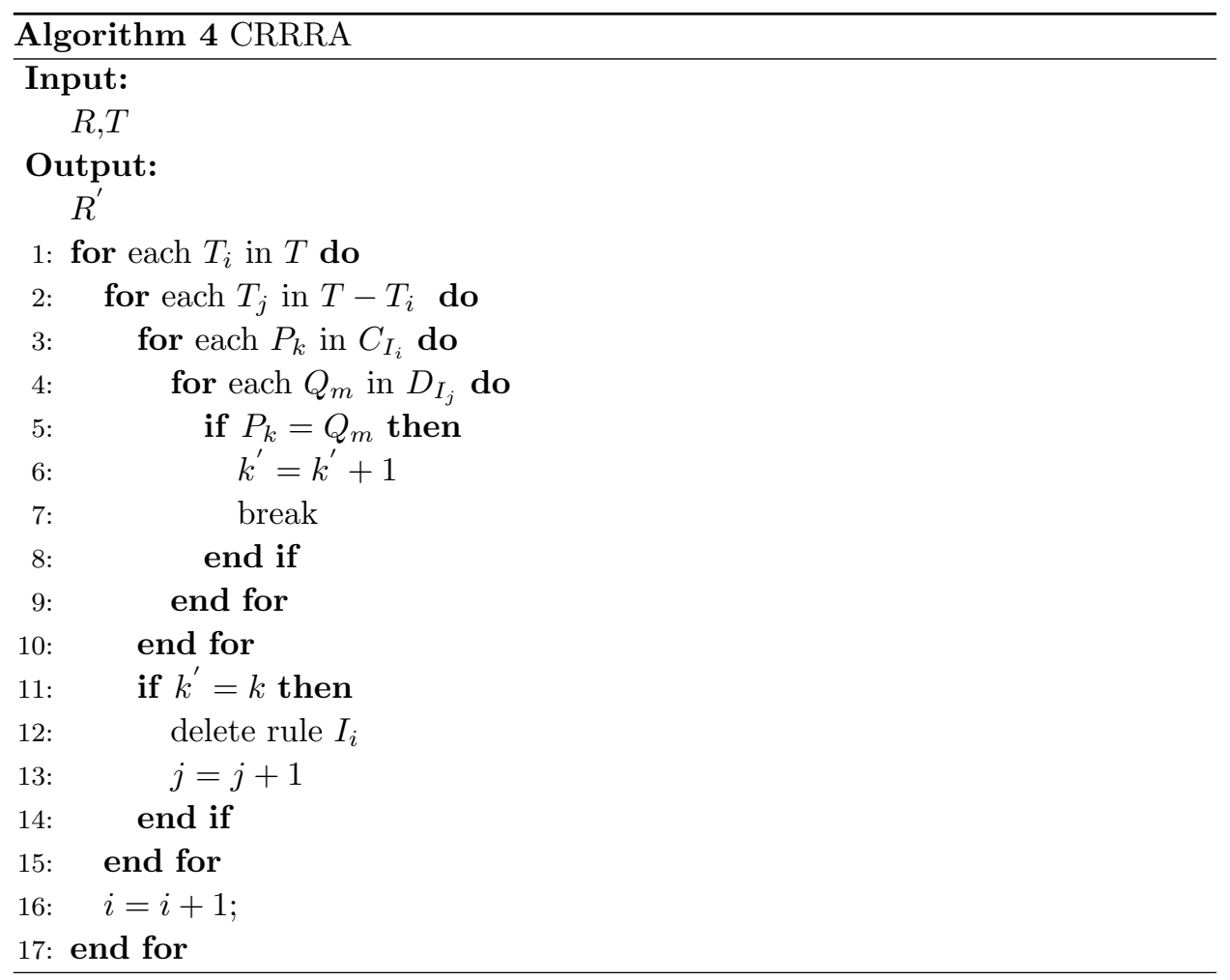


The new structure of premises is defined as $C_{i}^{\prime}$. And the new structure of conclusions is defined as $D_{j}^{\prime}$.

The $C_{i}^{\prime}$ is defined as ( $C_{k}$, cfrequency, cposition). The $C_{k}$ is the premise we define before. The cfrequency is the frequency of the $C_{k}$ in all the rules. Then cposition is an array with the length of cfrequency. And the cposition can be defined as $\left(\operatorname{cpos}_{1}, \operatorname{cpos}_{2}, \ldots, \operatorname{cpos}_{\text {frequency }}\right)$. The $\operatorname{cpos}_{m}$ is the $m$ rules position of $C_{k}$.

The $D_{j}^{\prime}$ is defined as ( $D_{k}$,dfrequency,dposition). The $D_{k}$ is the premise we define before. The dfrequency is the frequency of the $D_{k}$ in all the rules. The dposition is an array with the length of dposition. And the dposition can be defined as $\left(\operatorname{dpos}_{1}, \mathrm{dpos}_{2}, \ldots, \mathrm{dpos}_{\text {frequency }}\right)$. The $\operatorname{dpos}_{n}$ is the position in which there is $D_{k}$.

By the definition of premise all the premises can be described as a set $C^{\prime}$, the form of set $C^{\prime}$ is defined as the following way:

$$
C^{\prime}=\left\{C_{1}^{\prime}, \ldots, C_{r_{1}}^{\prime}\right\}
$$

And use the same way, the conclusions set can be denoted as follows:

$$
D^{\prime}=\left\{D_{1}^{\prime}, \ldots, D_{r_{2}}^{\prime}\right\}
$$

With this definition above, the $R_{\text {cycle }}$ can be defined as the set of all the cycle redundancy rules. The $R_{\text {cycle }}$ consist of links of rules. These links are cycle redundancy rules. These links are defined as $R_{\text {cycle }_{k}}$.

That means the $R_{\text {cycle }}$ can be described as the following:

$$
R_{\text {cycle }}=\left\{R_{\text {cycle }_{1}}, R_{\text {cycle }_{2}}, \ldots, R_{\text {cycle }_{n}}\right\} .
$$

And the $R_{\text {cycle }_{k}}$ can be described as the following:

$$
R_{\text {cycle }_{k}}=\left\{R_{1}, R_{2}, \ldots, R_{j}\right\} .
$$

The $R_{j}$ is the $j$ rule in the rule base. And the $R_{j}$ in $R_{\text {cycle }_{k}}$ can be defined as the following:

$$
R_{j}=\{C, D\}
$$

The $C$ is the premises set of $j$ rule. And the $D$ is the conclusions set of $j$ rule.

In order to do the reduction of cycle redundancy rules, the rule cycles in the rule should be find first base. So the cycles searching algorithm (CSA, Algorithm 5) can be presented here.

The ninth step judges whether the conclusions of $R_{k}$ are equal to the premises of $R_{\mathrm{pos}}$. In order to realize this function, an algorithm is conducted. This algorithm is present as equality judgment algorithm (EJA, Algorithm 6). In this algorithm every premise of $R_{\text {pos }}$ is compared with every conclusion of $R_{k}$. If the conclusions of $R_{k}$ are equal to the premises of $R_{\text {pos }}$, the tag is assigned with 1 . Otherwise it is assigned with 0 .

There are two functions in first step in Algorithm 6. The first function is defined as the number of conclusions of one rule. And the second function is defined as the number of premises of one rule. And this function can be easily realized by traveling 


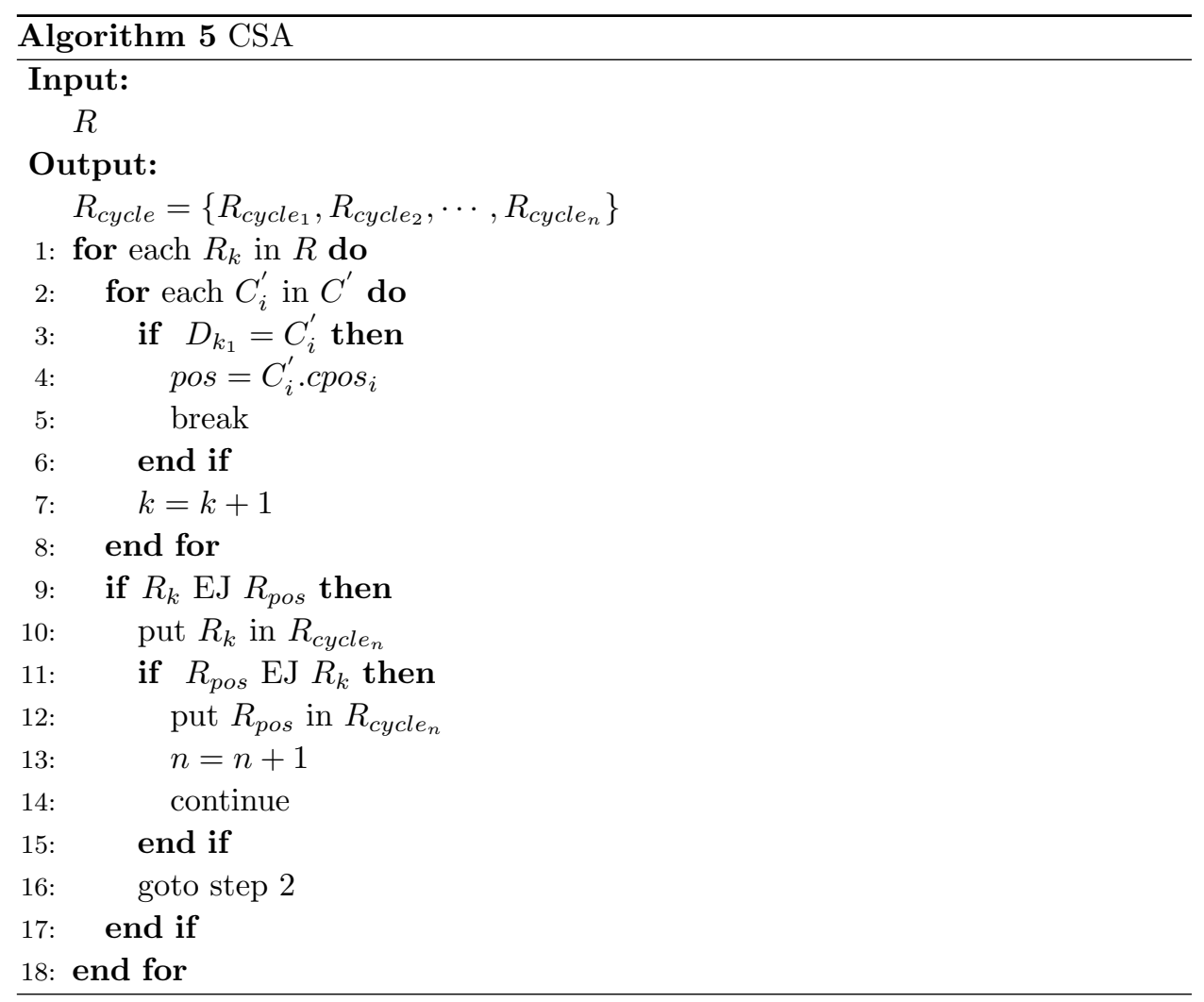

every premise or conclusion of one rule. So the explanation of these two functions are not introduced here. The function of these two algorithms is directly given here.

After finding the cycles in the rule base, an algorithm can be conducted to delete some of the rules in these cycles. It is easy to know that the rules in the cycle are redundant to this cycle, but these rules may be relevant to other rules which are not in this cycle. So it is not right to easily delete all the rules in the cycle. We need to judge whether the rules in the cycle is relevant to other rules which are not in this cycle as well.

So it is necessary to construct an algorithm for finding the premises and conclusions whose frequency is one in all of the rules. If the premises and conclusions of one rule only appear in this only rule, then this rule is irrelevant to the rules out of the cycle. The algorithm can be conducted to find the premises and conclusions whose frequency is one (FFOA, Algorithm 7).

The rules that are only relevant to the cycle are called as total cycle redundancy rules. And the algorithm can be conducted to find the total cycle redundancy rules and delete them. The algorithm is the deleting total cycle redundancy rules algorithm (DTCA, Algorithm 8). 




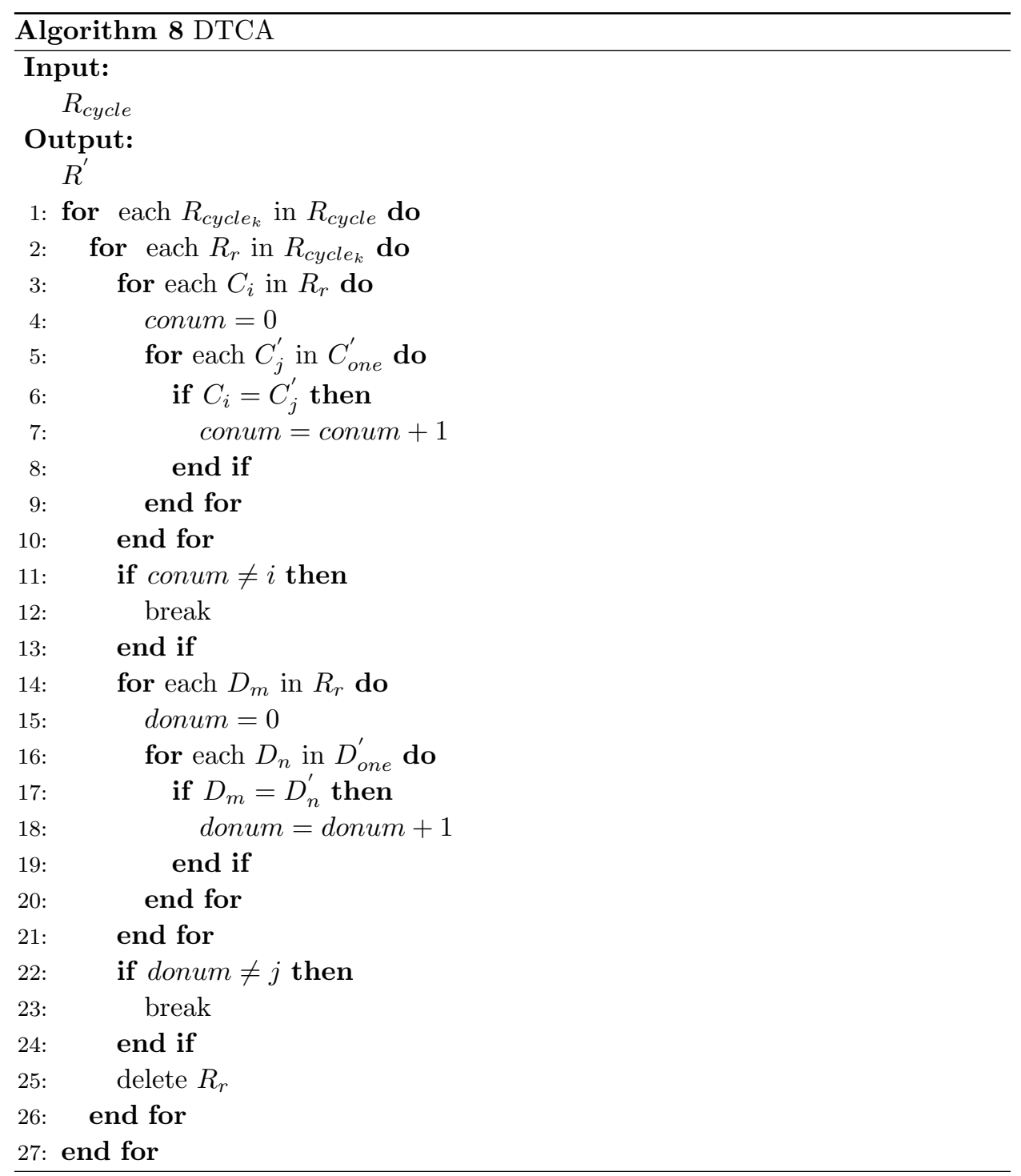

\section{Analysis for Reduction Algorithms}

The reduction algorithms for equivalent redundancy rules and implication redundancy rules use a virtual sorting before the reduction. This is a new idea of reduction for rule-based knowledge base. This method can avoid unnecessary matching to decrease the time and space of reduction for redundancy rules in the knowledge base.

The number of the propositions in a rule is far less than the number of the rules in a knowledge base. So when discussing the complexity of the algorithm, the number of the proposition is always not concerned. Without the virtual sorting in these two reduction algorithms, for the scale of $n$ the time complexity is $O\left(n^{2}\right)$. When applying 
the algorithm for the equivalent redundancy rules and implication redundancy rules and with the scale of $n$, every rule will be judged whether it should be reducted. And when judging one rule every rules in the rule base should be compared with that rule. So the time complexity is $O\left(n^{2}\right)$. But with the virtual sorting before the reduction algorithm for the same scale the time complexity is reduced. Let $a_{i}$ be the rule whose number of proposition is more than the number of the rule. When judging one rule not every rule in the rule base should be compared with that rule. Only the rule whose number of proposition is more than the number of the rule should be compared. And then the complexity is reduced to $\sum_{1}^{n} a_{i} * n$. When the number of the rules is huge, this algorithm is superior to the algorithm without virtual sorting.

And the reduction algorithm for cycle redundancy rules is successfully conducted. After the reduction for cycle redundancy rules, the reasoning in the rule-based knowledge base will not fall into the reasoning loop. And the time complexity of the reduction algorithm for the cycle redundancy rules is $O\left(n^{2}\right)$.

\section{Conclusion}

This paper presents the notions of redundancy rules, and gives the classification of redundancy rules. By all of these notions of rules and rule-based knowledge base there is a virtual sorting conducted to decrease the time complexity.

And there are the reduction algorithms of four kinds of redundancy rules. These types of redundancy rules are equivalent redundancy rules, implication redundancy rules and cycle redundancy rules in explicit redundancy rules and condition redundancy rules in implicit redundancy rules.

The equivalent redundancy rules and condition redundancy rules are presented with the virtual sorting algorithm. With the virtual sorting algorithm the time complexity of these two algorithms can be reduced from $O\left(n^{2}\right)$ to $\sum_{1}^{n} a_{i} * n$. And the time complexity of the reduction algorithm for the cycle redundancy rules is $O\left(n^{2}\right)$.

The research for the redundancy rule is a meaningful work. Meanwhile there processing about the uncertain data is becoming a hot issue, see Refs. 14 and 15 for examples. So the reduction for redundancy rules over the uncertain data should be the focus of our future work.

\section{Acknowledgments}

This work is supported by the National Natural Science Foundation of China (61272171, 61371090) and the Fundamental Research Funds for the Central Universities of China (31320156306).

\section{References}

1. L. An and J. Wang, Research on the redundancy testing method for the rule base of expert system, Comput. Eng. Appl. 44(34) (2008) 191-193.

2. Z. Ao, Artificial Intelligence and Expert System. (Machine Press, Beijing, 2010). 
3. A. Chandanan and M. Shukla, Removal of duplicate rules for association rule mining from multilevel dataset, Procedia Comput. Sci. 45(2) (2015) 143-149.

4. M. Guo, H. Sun and T. Huang, Maintenance of knowledge base in expert system, High Technol. Lett. 12(2) (2002) 1-4.

5. W. Huang and J. Yang, Maintenance of knowledge base in expert system, Comput. Age (1) (2005) 40-41.

6. X. Jia, L. Song, R. Wang and W. Li, Rule bases mering and redundancy, circularity and conflict detection, Comput. Appl. Softw. 33(1) (2016) 249-253.

7. X. Jiang and J. Wang, Design and implement of grid service for resolving rules inconsistency, Electron. Sci. Technol. 26(12) (2013) 138-140.

8. R. Li, R. Perneczky, A. Drzezga and S. Kramer, Efficient redundancy reduced subgroup discovery via quadratic programming," J. Intell. Inf. Syst. 44(2) (2015) 271-288.

9. S. Luan and G. Dai, An algebraic method for the updating of proposition rule-based knowledge base, Sci. China 38(2) (2008) 177-194.

10. R. Miao and X. Shen, The extraction algorithm of concept lattice in non redundancy association rules, Comput. Eng. 22(35) (2009) 74-76.

11. D. Theodoratos and T. Sellis, Designing data warehouses, Data and Knowledge Engineering 31(3) (1999) 279-301.

12. M. Unde and R. Simran, A novel technique for effective optimization of cross domain network protocol for redundancy removal in firewall policies, Int. J. Comput. Appl. $\mathbf{1 2 2}(22)(2015)$ 16-21.

13. X. Zhao, Redundancy test of rule base, Comput. Dev. Appl. 19(12) (2006) 6-11.

14. X. Zhou, K. Li, G. Xiao and Y. Zhou, Top k favorite probabilistic products queries, IEEE Trans, Knowl. Data Eng. 28(10) (2016) 2808-2821.

15. X. Zhou, K. Li, Y. Zhou and K. Li, Adaptive processing for distributed skyline queries over uncertain data, IEEE Trans. Knowl. Data Eng. 28(2) (2016) 371-384. 


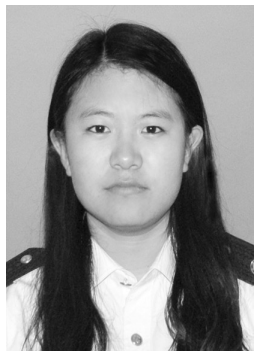

Yongjie Zhang was born in May 1992, and raised in Baoding city, Hebei province of China. She graduated from Dalian Maritime University in 2014. She has been working on her masters degree in Dalian Maritime University since 2014. Her research is in the areas of artificial intelligence and computer algorithm.

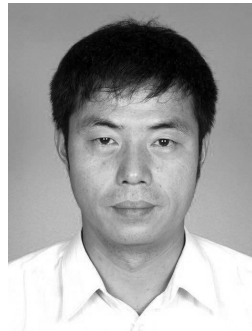

Ansheng Deng was born in June 1963, and raised in Jiaohe city, Jilin Province, P. R. China. He received his B.S. degree from Beijing University in 1985, his M.S. degree and Ph.D. in Computer Science from Jilin University in 1988 and 1995, respectively. He was with the Department of Computer Science at Northeast Normal University from June 1988 to May 2001, and the School of Information Science and Technology at Xiamen University from June 2001 to July 2005, then joined the School of Computer and Information Technology at Beijing Jiaotong University until December 2006. Currently he is a professor at the School of Information Science and Technology of Dalian Maritime University. Dr. Deng's research is in the areas of artificial intelligence and computer algorithm. His research deals mainly with logic foundations of artificial intelligence and automated reasoning in classical or non-classical logic. He has been involved with 8 projects supported by the National Natural Science Foundation of China, and with 2 projects supported by the National High Technique Developing Program. He has published over 60 research papers. 